\title{
Analytic steady-state space use patterns and rapid computations in mechanistic home range analysis
}

\author{
Alex H. Barnett \\ Department of Mathematics, 6188 Kemeny Hall, Dartmouth College, Hanover NH 03755, USA* \\ Paul R. Moorcroft \\ OEB Dept, Harvard University, 22 Divinity Ave, Cambridge MA 02138, USA
}

(Dated: October 24, 2018)

\begin{abstract}
Mechanistic home range models are important tools in modeling animal dynamics in spatiallycomplex environments. We introduce a class of stochastic models for animal movement in a habitat of varying preference. Such models interpolate between spatially-implicit resource selection analysis (RSA) and advection-diffusion models, possessing these two models as limiting cases. We find a closed-form solution for the steady-state (equilibrium) probability distribution $u^{*}$ using a factorization of the redistribution operator into symmetric and diagonal parts. How space use is controlled by the preference function $w$ then depends on the characteristic width of the redistribution kernel: when $w$ changes rapidly compared to this width, $u^{*} \propto w$, whereas on global scales large compared to this width, $u^{*} \propto w^{2}$. We analyse the behavior at discontinuities in $w$ which occur at habitat type boundaries. We simulate the dynamics of space use given two-dimensional prey-availability data and explore the effect of the redistribution kernel width. Our factorization allows such numerical simulations to be done extremely fast; we expect this to aid the computationally-intensive task of model parameter fitting and inverse modeling.
\end{abstract}

\section{INTRODUCTION}

Due to the influences of habitats on the availability of food, shelter, mates and risk of predation, patterns of animal space use are strongly influenced by the spatial distribution of habitat types across landscapes. A widespread technique for analyzing such relationships between habitats and patterns of animal space use has been resource selection analysis (RSA) [1, 4, 7], in which the intensity of space use is assumed to reflect an underlying resource selection function giving an individual's preference for the habitat type found at that location. However, implicit in this approach is an assumption that all habitats are equally accessible to an individual regardless of its current position: no account is made of the individual's finite movement speed or the spatial geometry of habitats on the landscape on which it moves $[10,11]$.

More recently, an alternative framework has emerged in the form of mechanistic home range models [6, 11, 12]. In contrast to RSA which is largely descriptive, such models yield spatially-explicit predictions for patterns of animal space use in the form of a probability density function (pdf), by modeling the process of individual movement. Mathematically, the fine-scale behavior of individuals is treated as a stochastic (Markov) process [5, 13, 15, 17], specifying the probability of an animal at a given location moving to a subsequent location during a given time interval. From this description one can derive, in the limit of small time intervals, a continuous-time partial differential equation (PDE) for the evolution of the pdf. For example, a recent analysis of coyote home ranges in Yellowstone 12] used a "prey availability plus conspecific avoidance" (PA+CA) mechanistic home range model to account for the observed patterns of coyote home ranges within the park. In this model, individuals exhibited an avoidance response to encounters with foreign scent marks and a foraging response to prey availability (individuals decreased their mean step length in response to increasing small mammal abundance in different habitats.) In such mechanistic models, inferences about long-term space use are usually made by evolving the continuous-time PDE in order to converge to its steady-state; this can be computationally time-consuming [9, 11].

In recent work [10], we developed a mechanistic home range model which reconciles these two main approaches by combining the concept of a spatial preference function $w(x)$ with a stochastic model of fine-scale movement behavior. At each time-step the movement of an individual is governed by its relative preference for the local habitat surrounding its current spatial position i.e. the preference function restricted to a region of size $L$ centered on the individual's current location. The length scale $L$ has two roles: it is the typical (jump) distance per time step, but also can be interpreted as the distance over which the animal is able to perceive differences in surrounding habitats. This new

*Electronic address: ahb@math.dartmouth.edu 
a) RSA

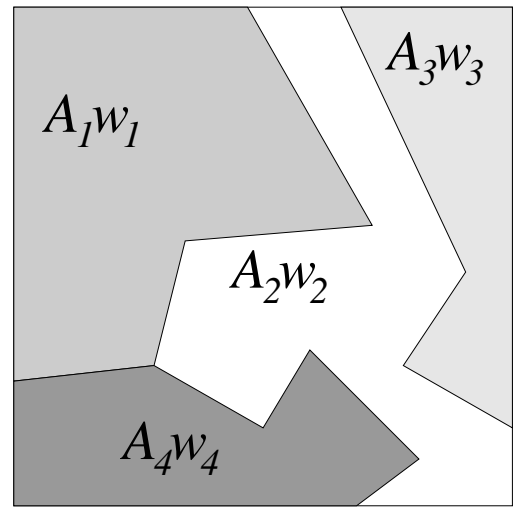

b) Proposed mechanistic model

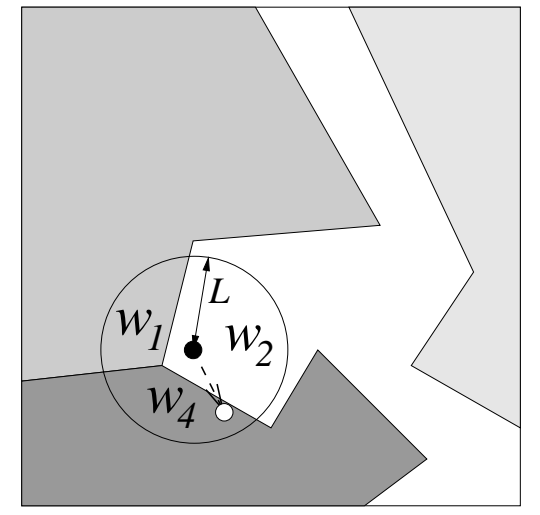

FIG. 1: Schematic comparing conventional resource selection analysis (RSA) against our proposed model for space use, in two dimensions. Shaded regions show areas of habitat in which the preference function $w(x)$ is constant. a) RSA. The probability of finding an animal in each region is proportional to the product of preference (resource selection function) $w_{j}$ and area of the region $A_{j}$. There is no account taken of the animal's current habitat type or location within the habitat. b) Proposed MERSA model for space use. The animal responds only to the preference function within a distance scale $L$ from its current location (black dot). Each time step the future location (white dot) is chosen randomly from a localized distribution biased by the preference function.

model, which we will call a MEchanistic RSA, or MERSA model, is compared against traditional RSA in Fig. 1 and detailed in Section [II] In [10] we were able to show that, in one dimension (1D) with a spatially smooth habitat preference function, using the Kramers-Moyal expansion ([2, 10] and App. A and E of [11]) one may derive an an advection-diffusion equation for the expected patterns of space use, with advection and diffusion coefficients related to the parameters of the underlying stochastic movement model. We then showed that the resulting steady-state pdf could be determined analytically, giving a intensity of space use proportional to the square of the preference function.

In this paper we expand and generalize this result to the more biologically relevant case of individuals moving in two space dimensions across landscapes which may include discrete habitat types giving rise to a non-smooth preference function. Specifically, in Section [II] we solve for the steady-state pdf of our discrete-time stochastic model of fine-scale movement behavior directly and analytically, for arbitrary preference functions, without resorting to the conventional procedure of taking the continuous-time limit [19]. (Note that this relies on a particular algebraic feature of our model; for a general redistribution kernel such an analytic solution is not available.) By doing so, we are able rapidly to compute exact steady-state space use patterns for the model for a full range of possible length scale values $L$, rather than being confined to the limit of small $L$ inherent in a PDE-based approach. We also gain an understanding of the numerically-observed pdf behavior at jumps in preference function, which had remained a mystery [10]. A consequence of our solution is that the computationally-intenstive task of solving an inverse problem to fit multiple model parameters can become orders of magnitude faster. We discuss such numerical implications in Section IIA. and give CPU timings for our numerical examples throughout.

For illustrative purposes, we choose a translationally-invariant exponential distribution of jump lengths, a kernel which has proven useful for modeling coyote foraging movement [11]. However, our analytic solution also holds for a generalization of the model of [10] to spatially-varying diffusion coefficient (spatially-dependent length scale $L$ ) such as occurs in modeling the prey-density dependent foraging rate of wolves and coyotes [6, 12, 18]. In Section [IVA we explore the transition from small $L$ (where the model tends to the advection-diffusion equation derived in [10]), to large $L$ (where the model becomes equivalent to RSA). Here 'large' and 'small' are in comparison to the typical spatial scale on which the preference function changes. In Section IVA we explore this numerically both in $1 \mathrm{D}$, and with a 2D preference function derived from discontinuous real-world small mammal abundance data appropriate for coyotes. In Section IVB we show rapid and efficient numerical simulation of the time evolution of the pdf. Furthermore in Section V we analyse the behavior at a sharp discontinuity in preference function, and show that on spatial scales larger than $L$ this effect may be approximated by effective matching conditions in coupled advection-diffusion equations. Finally in Section [V1] we discuss implications and draw conclusions. 


\section{MECHANISTIC SPATIALLY-EXPLICIT (MERSA) MODEL}

The time-dependent pdf of an animal we will represent by $u(x, t)$, where $x \in \Omega$ is the location, $\Omega \subset \mathbb{R}^{d}$ represents the habitat region of interest, and $d$ is the dimensionality of space (usually 1 or 2 ). Thus, in a $1 \mathrm{D}$ setting, $u(x, t) d x$ is the probability that at time $t$ a given individual is to be found in the interval $[x, x+d x]$. (Note in $2 \mathrm{D}$ we use $x$ rather than $\boldsymbol{x}$ to represent location vector.) Its normalization is

$$
\int_{\Omega} u(x, t) d x=1 \quad \text { for all } t
$$

Our MERSA model is an example of a Markov process. At each time step of length $\tau$ the current pdf at time $t$ is acted on by a fixed linear operator to get the pdf at time $t+\tau$. This is expressed by the master equation

$$
u(x, t+\tau)=\int_{\Omega} k\left(x, x^{\prime}\right) u\left(x^{\prime}, t\right) d x^{\prime} \quad \text { for } x \in \Omega
$$

Given an initial pdf $u(x, 0)=u_{0}(x)$ for all $x \in \Omega$, by iteration the pdf at arbitrarily large future times (multiples of $\tau$ ) may be computed. Here the redistribution operator kernel $k\left(x, x^{\prime}\right)$ is defined as the conditional pdf of an individual animal's location $x$, a time interval $\tau$ into the future, given that its current location is $x^{\prime}[20$ ]. This is an uncorrelated jump or 'kangaroo' process [14] (note the animal has no memory beyond the fixed time scale $\tau$ ). Since a redistribution kernel is a conditional pdf it is everywhere non-negative and ('columnwise') normalized by

$$
\int_{\Omega} k\left(x, x^{\prime}\right) d x=1 \quad \text { for all } x^{\prime} \in \Omega
$$

Clearly for a given ecological situation the choice of $\tau$ determines the form of $k\left(x, x^{\prime}\right)$. For example, shorter $\tau$ may demand a smaller kernel width $L$ simply because animal speed is limited. By the length scale $L$ we mean the typical size of $\left|x-x^{\prime}\right|$ for which $k\left(x, x^{\prime}\right)$ is significant. We will not indicate explicitly the dependence on $\tau$ of the form of $k$. The appropriate value of $\tau$ depends on the application; it needs to be large enough that successive animal relocations can be approximately treated as uncorrelated. Real-world location data collection technology also can be a factor if fine-scale model fitting is to be done. For coyotes, a typical value of $\tau$ is 10 minutes [11].

We consider a spatial preference (resource selection) function $w \in L^{1}(\Omega)$ which controls relative preference for each location in the domain. We represent unbiased ('preference-free') diffusive animal movement with a symmetric redistribution kernel $\phi\left(x, x^{\prime}\right)$, that is,

$$
\phi\left(x, x^{\prime}\right)=\phi\left(x^{\prime}, x\right) \quad \text { for all } x, x^{\prime} \in \Omega .
$$

The kernel $\phi$ obeys the Markov normalization (3); from this and symmetry it follows that

$$
\int_{\Omega} \phi\left(x, x^{\prime}\right) d x^{\prime}=1 \quad \text { for all } x \in \Omega
$$

which is the statement that a constant pdf is invariant under redistribution by $\phi$. Since a uniform density gives no net probability mass flow, we say that (an operator with kernel) $\phi$ is advection-free. Our MERSA redistribution kernel $k$ is this advection-free jump kernel biased by the preference function, in other words,

$$
k\left(x, x^{\prime}\right)=\frac{w(x) \phi\left(x, x^{\prime}\right)}{z\left(x^{\prime}\right)}
$$

with normalization function (easily seen to be required to satisfy (30)),

$$
z\left(x^{\prime}\right):=\int_{\Omega} w\left(x^{\prime \prime}\right) \phi\left(x^{\prime \prime}, x^{\prime}\right) d x^{\prime \prime} .
$$

For (6) to be meaningful we must have $z>0$ everywhere; it is sufficient that $w>0$ everywhere for this to hold, which we will assume from now on. Note that since $\phi\left(x, x^{\prime}\right)$ may depend independently on $x$ and $x^{\prime}$ (barring the symmetry constraint), it may represent a spatially-varying (and also anisotropic) diffusion coefficient. The MERSA model is thus more general than that of [10], which was restricted to the translationally-invariant case

$$
\phi\left(x, x^{\prime}\right)=\tilde{\phi}\left(x-x^{\prime}\right) .
$$

Here $\tilde{\phi}(\cdot)$ is a function of relative displacement alone, which limits one to a spatially-invariant distribution of steplengths in the underlying stochastic movement model. 


\section{A. Limiting cases of the model}

We now discuss two limiting forms of MERSA. Firstly, consider the case $\phi\left(x, x^{\prime}\right)=1 / \operatorname{vol}(\Omega)$ for all $x, x^{\prime} \in \Omega$. This corresponds to preference-free redistribution to a uniformly-random location in $\Omega$, without regard to current location. (6) then becomes

$$
k\left(x, x^{\prime}\right)=\frac{w(x)}{\int_{\Omega} w\left(x^{\prime \prime}\right) d x^{\prime \prime}} .
$$

Since this is independent of $x^{\prime}$, within a single time step (and for all future time steps) the master equation reaches its steady-state pdf $u^{*}:=\lim _{t \rightarrow \infty} u(\cdot, t)$ given by

$$
u^{*}(x)=C_{1} w(x), \quad \text { with normalization constant } \quad C_{1}^{-1}=\int_{\Omega} w\left(x^{\prime \prime}\right) d x^{\prime \prime} .
$$

This is formally equivalent to a conventional (time-invariant) RSA model, with linear dependence on preference. This assertion is illustrated when $\Omega$ is divided into regions $j=1 \cdots m$ each of area $A_{j}$ and constant preference function $w_{j}$, for then (10) assumes the more familiar RSA form $u_{j}=A_{j} w_{j} /\left(\sum_{i=1}^{m} A_{k} w_{k}\right)$ where $u_{j}$ is the probability of being in region $j$ [1, 4, 7], see Fig. 17. In the case of an infinite domain such as $\Omega=\mathbb{R}^{d}$ (in which case $w$ alone delineates the habitat) no constant normalizable $\phi$ exists; however, the above result may be reproduced by considering the limit in which a the kernel $\phi$ becomes much wider than all spatial scales of interest in the habitat $(L \rightarrow \infty)$. Then $\phi\left(x, x^{\prime}\right)$ tends to a constant for $x$ and $x^{\prime}$ within the habitat, and (10) again follows. Thus for both cases above we will call this the $L \rightarrow \infty$ limit, and state that in this limit our MERSA model degenerates to RSA.

Secondly, consider the $L \rightarrow 0$ limit where $k$ tends to a diagonal kernel. In order for time evolution to take place at all, we must also take the limit $\tau \rightarrow 0$. It is well known [13, 14] that the correct way to balance these two limits in order to reach a well-defined diffusion coefficient is to choose the variance of the kernel $k$ to scale as $\tau$. In the 1D case of smooth $w$, and a translation-invariant kernel (8), we have derived [10] that in this limit our MERSA model gives a Fokker-Planck PDE with known advection and diffusion coefficients. From this we showed that the steady-state pdf is

$$
u^{*}(x)=C_{2} w^{2}(x), \quad \text { with constant } \quad C_{2}^{-1}=\int_{\Omega} w^{2}\left(x^{\prime \prime}\right) d x^{\prime \prime},
$$

that is, quadratic in preference function. (The integral is bounded since $w$ is smooth and in $L^{1}(\Omega)$ ). We note that in this Fokker-Planck limit, our model is equivalent to that of [3] with the 'potential' function $U(x)=-2 D \log w(x)$ and constant diffusion $d(x)=D$.

We will see in Section IVA how these differing $L \rightarrow \infty$ and $L \rightarrow 0$ steady-state limits are reached in practice.

\section{ANALYTIC FORMULA FOR STEADY-STATE PDF}

The condition that $u^{*}$ be a steady-state pdf is that it be invariant under the master equation (2), in other words,

$$
u^{*}(x)=\int_{\Omega} k\left(x, x^{\prime}\right) u^{*}\left(x^{\prime}\right) d x^{\prime} \quad \text { for all } x \in \Omega .
$$

Our main result is the following claim.

Proposition 1 A steady-state pdf for the model redistribution kernel (6) is given by

$$
u^{*}(x)=C w(x) z(x), \quad \text { with constant } \quad C^{-1}=\int_{\Omega} w\left(x^{\prime}\right) z\left(x^{\prime}\right) d x^{\prime},
$$

where the function $z$ is defined by (7).

The proposition is proved by substituting (6) and (13) into the right side of (12) then noticing that $z$ cancels, allowing the simplification

$$
\begin{aligned}
\int_{\Omega} k\left(x, x^{\prime}\right) C w\left(x^{\prime}\right) z\left(x^{\prime}\right) d x^{\prime} & =C \int_{\Omega} w(x) \phi\left(x, x^{\prime}\right) w\left(x^{\prime}\right) d x^{\prime} \\
& =C w(x) \int_{\Omega} \phi\left(x^{\prime}, x\right) w\left(x^{\prime}\right) d x^{\prime} \\
& =C w(x) z(x), \\
& =u^{*}(x),
\end{aligned}
$$


verifying (12). Crucially, it is the assumption that $\phi$ is symmetric that allows us to proceed from the first to second line. Notice that once standard assumptions about ergodicity are satisfied, the steady-state $u^{*}$ is unique $(e . g$. see Doeblin condition in [8] p.396; in our ecological context this is satisfied since we can assume that there is always some randomness to animal motion, i.e. the kernel $\phi$ is always somewhat spreading at each spatial location). Since $w$ is assumed to be everywhere positive, so is $u^{*}$.

We now derive some secondary results on the structure of $K$, giving intuition into the reason for existence of the simple formula (13). They may be skipped if no further mathematical insight is required. We switch to operator notation, expressing (12) as $u^{*}=K u^{*}$ where $K$ is a Markov operator. Recall that a Markov operator is an integral operator with non-negative kernel obeying (3), which can be expressed $K^{T} 1=1$ where 1 is the constant function and $K^{T}$ the adjoint operator (with respect to uniform measure). Our model (6) expresses the factorization

$$
K=W \Phi Z^{-1}
$$

where $\Phi$ is the (Markov) operator defined by the integral kernel $\phi\left(x, x^{\prime}\right)$, and $W$ and $Z$ are the diagonal operators which multiply by the functions $w$ and $z$ respectively. Thus the structure is an operator with constant invariant measure sandwiched between two diagonal operators. Note that $z=\Phi w$ since $\Phi$ is symmetric. Then we have,

Proposition 2 With the assumption $w \geq c$ everywhere, for some $c>0$, our model Markov operator (15)

1. satisfies detailed balance, that is,

$$
k\left(x, x^{\prime}\right) u^{*}\left(x^{\prime}\right)=k\left(x^{\prime}, x\right) u^{*}(x) \quad \text { for all } x, x^{\prime} \in \Omega
$$

2. is self-adjoint with respect to the measure $1 / u^{*}$, and

3. has all eigenvalues real.

The proof is as follows. We define $U^{*}$ to be the operator multiplying by the function $u^{*}$; it can be written $U^{*}=Z W$. Using (15) gives $K U^{*}=W \Phi W$, explicitly symmetric. In other words

$$
\left(K U^{*}\right)^{T}=K U^{*}
$$

which is equivalent to detailed balance (16). Now we use $(\cdot, \cdot)$ to indicate the $L^{2}(\Omega)$ (real) inner product with respect to uniform measure, and $(\cdot, \cdot)_{1 / u^{*}}$ with respect to measure $1 / u^{*}$. Using (17) and the boundedness of $1 / u^{*}$ in the middle step we have

$$
(a, K b)_{1 / u^{*}}=\left(\frac{a}{u^{*}}, K U^{*} \frac{b}{u^{*}}\right)=\left(K U^{*} \frac{a}{u^{*}}, \frac{b}{u^{*}}\right)=(K a, b)_{1 / u^{*}} \quad \text { for all } a, b \in L^{2}(\Omega)
$$

which proves part 2. Part 3 immediately follows by self-adjointness. We remark that the simple formula (13) for $u^{*}$ can now be seen as a result of the need to symmetrize the factorization (15).

\section{A. Implications for fast numerical modeling}

We discuss briefly why the above result is important in practice. The analytic steady-state pdf formula (13) is special because, for a general redistribution kernel no such formula exists. In particular, there is no formula for $u^{*}$ for commonly-used mechanistic kernels such as those expressed in polar coordinates with exponential radial jumps and a von Mises angular distribution (Ch. 3 of [11]). (In that work all steady-state distributions had to be computed in the advection-diffusion limit, often in a numerically-intensive fashion; see App. G of [11] and [9]). In fitting model parameters to real-world location data, a large number of such steady-state pdfs must be found as part of the parameter-optimization process (e.g. Sec. 4.3 of [11]).

Numerical solution of $u^{*}$ for any redistribution kernel requires discretization of the domain into $N$ degrees of freedom. In $2 \mathrm{D}$ the $N$ required for acceptable accuracy can be large $\left(e . g .10^{4}\right)$. Solving for $u^{*}$ given a general kernel is then an eigenvector problem involving a (possibly dense) $N$-by- $N$ matrix discretization of that kernel. The iterative solution of such large eigenproblems can be slow especially when diffusion rates are small. In constrast, the formula (13) in our MERSA model bypasses this and requires only the computational effort of the single matrix-vector multiplication required to evaluate the (discretized) integral (7). This is an acceleration by orders of magnitude.

There is a further numerical advantage to a special case of the MERSA model. Namely if $\phi$ is translationallyinvariant (diffusion coeffcient is spatially constant) then the action of the (discretized) convolution operator $\Phi$ may 

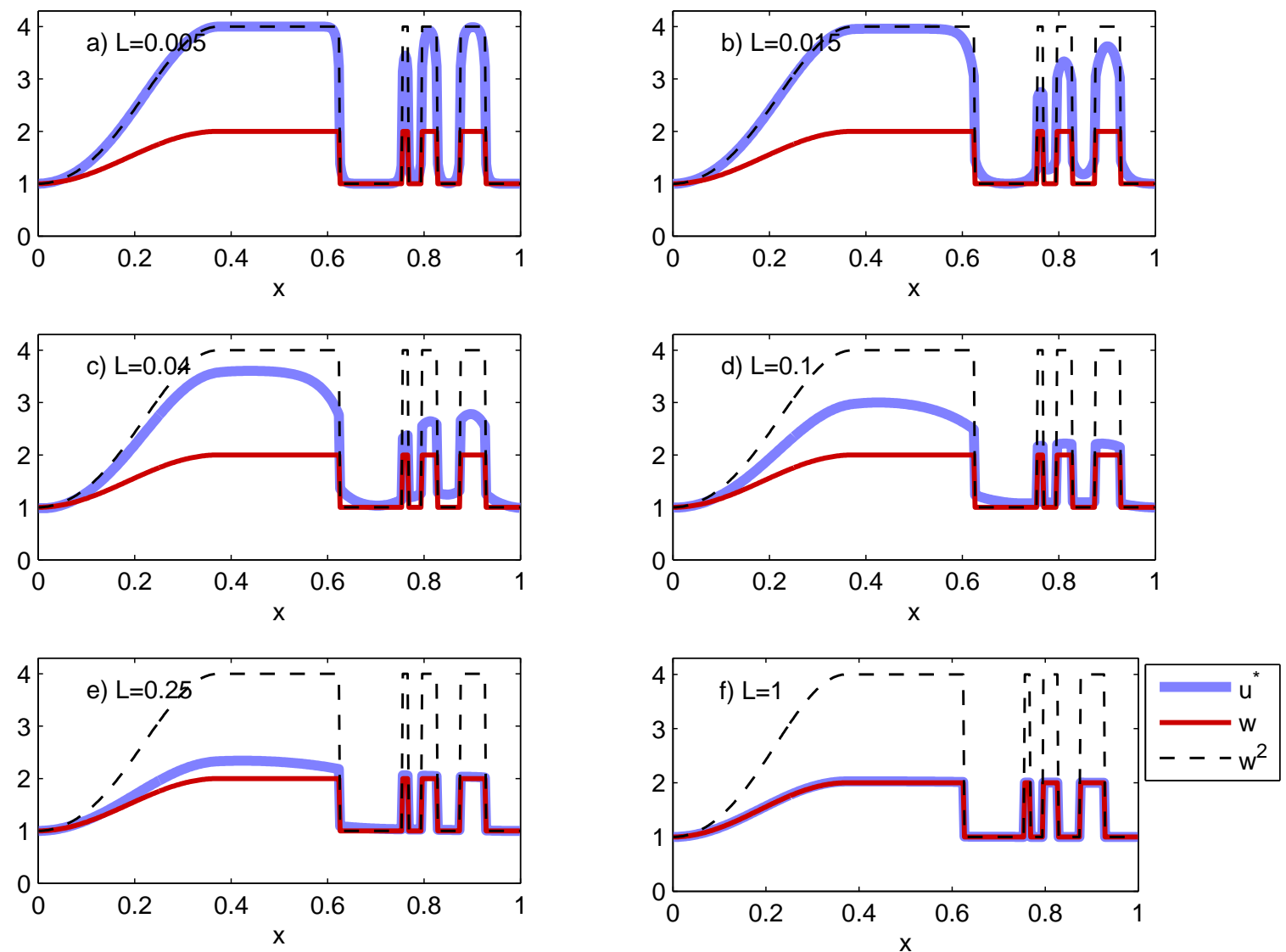

FIG. 2: Steady-state pdfs $u^{*}$ for exponential jump pdf of (19) for an increasing sequence of $L$ values. The preference function $w$ (thin solid line) is chosen to be smooth on the left side of the domain, and discontinuous and oscillatory on the right side.

be computed in time $O(N \ln N)$ via the Fast Fourier Transform (FFT) [16], which for large $N$ is much faster than the $O\left(N^{2}\right)$ dense matrix-vector multiply. This further speeds up computing $u^{*}$ via (77). Finally, the time-evolution $u(x, t)$ can now be computed much more efficiently. A single time-step of the master equation (2) may be performed with $O(N \ln N)$ effort by using (15) in a split-operator method: division by $z$, followed by FFT application of $\Phi$, followed by multiplication by $w$.

We illustrate these numerical techniques and advantages below. All CPU timings are reported using a single core of a $2 \mathrm{GHz}$ Intel Core Duo processor running MATLAB 7.0 in GNU/Linux.

\section{NUMERICAL RESULTS FOR AN EXPONENTIAL JUMP KERNEL}

In this section we illustrate the predictions of our MERSA model for steady-state and time evolution of space use for an idealized 1D preference function, and for a 2D preference function that is based on spatially-complex, real-world measurements of prey abundance in different habitat types. Throughout we consider a translationally-invariant $\phi$ kernel, choosing an exponential kernel with width $L$, which takes the form in 1D

$$
\tilde{\phi}(\rho)=\frac{1}{2 L} e^{-|\rho| / L}
$$

where $\rho:=x-x^{\prime}$, and in $2 \mathrm{D}$

$$
\tilde{\phi}(\rho)=\phi(|\rho|)=\frac{1}{2 \pi L} \frac{e^{-|\rho| / L}}{|\rho|}
$$

where we remind the reader that $\rho$ is a vector in the latter. This $2 \mathrm{D}$ kernel has been used as a model of animal movement, for instance describing the fine-scale movement of coyotes with remarkable accuracy [11]. Note that the 


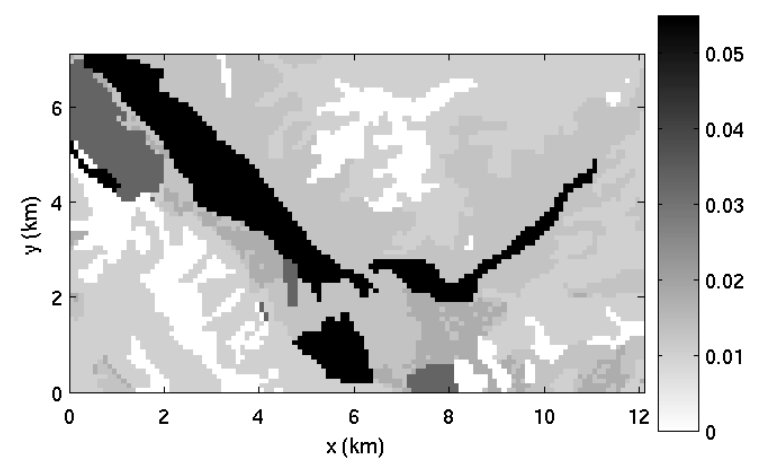

FIG. 3: Small-mammal abundance for Lamar Valley region of Yellowstone National Park collected by Crabtree et. al. (unpublished); see Ch. 7 of [11]. Units are the total biomass density of small mammals in $\mathrm{kg} / \mathrm{ha}$ with darker colors indicating larger values. The $x$ - and $y$-axes are in kilometers. The highest abundances of small mammals are found in the mesic grassland habitats.

2D kernel is radially symmetric, and when integrated over angle it gives a distribution of jump distances $r:=|\rho|$ which is exponential, $(1 / L) e^{-r / L}$.

\section{A. Steady-state}

Here we will assume the domain has periodic boundary conditions. It has the interpretation that the piece of habitat $\Omega$ in question is surrounded by similar (repeating) habitat, often a reasonable assumption. Mathematically this condition is achieved in the case of the unit interval $\Omega=[0,1)$ by replacing (19) by a sum over a few nearby 'image' kernels,

$$
\tilde{\phi}(\rho)=\frac{1}{2 L} \sum_{m=-M}^{M} e^{-|\rho-m| / L}
$$

where $M$ is chosen such that $\tilde{\phi}$ is periodic to some high accuracy $\left(e . g \cdot 10^{-6}\right)$. An analogous $2 \mathrm{D}$ image sum is used in the $2 \mathrm{D}$ case where $\Omega$ is a rectangle.

Note that our formalism can handle other boundary conditions. For instance we model a standard zero-flux boundary in Section IVB. We have also checked that the periodic steady-state results are very similar to those for zero-flux, except when $L$ is of order the size of the whole domain. (In this case for zero-flux $u^{*}$ is affected by the effective boundary discontinuity in $w$, a complication which we will not pursue here).

\section{One-dimensional case}

In Fig. 20 we show $u^{*}$ computed via (13) and (7) for the exponential kernel, for an ascending sequence of $L$ values, in the unit interval. Our example preference function $w$ has been chosen to exhibit a variety of lengthscales: for $x<0.6$ it is smooth, corresponding to a gradation in habitat preference, whereas for $x>0.6$ it is piecewise constant with discontinuous oscillations between the values 1 and 2 corresponding to isolated patches of more favorable habitat. The shortest length scale of $w$ is 0.015 , namely the size of the smallest constant patch near $x=0.76$. We see that for very small $L, u^{*}$ accurately matches $w^{2}$ for all regions apart from those with the most rapid $w$ variations. This matches the expectation in Section $\amalg A$.

A gradual transition is seen in the sequence of Fig. 2, As $L$ becomes larger than a given feature, $u^{*}$ in the vicinity of that feature starts to become locally proportional to $w$. Finally in plot (f), $L$ is larger than any feature and $u^{*}$ globally becomes linear in $w$. The explanation for this transition is simple and lies with (13) combined with the realisation that the function $z$ is given by the function $w$ smoothed locally over a width of about $L$. Fine $(<L)$ features in $w$ will thus be smoothed away giving a locally constant $z$, whereas for coarse $(>L)$ features $z \approx w$ and quadratic dependence in $w$ results. This effect is common to any jump kernel with characteristic width $L$ : fine-scale habitat features result in $u^{*} \propto w$, in accordance with the $L \rightarrow \infty$ limit (10), whereas coarse-scale habitat features are tracked according to $u^{*} \propto w^{2}$, in accordance with the Fokker-Planck limit (11). 
Since $u^{*}$ is computed analytically via (13), its numerical accuracy is limited only by quadrature of the integral. We computed $z$ using (7) via FFT convolution (Section (IIIA) in a few thousandths of a second on a uniform quadrature grid of $N=400$ points. This contrasts the order $1 \mathrm{~s}$ needed to iteratively solve for the dominant eigenvector of the dense matrix discretization of $\Phi$ that would be required if no simplifying factorization of $K$ or analytic solution for $u^{*}$ were known.

\section{Two-dimensional case with real-world habitat heterogeneity}

We start with the small-mammal density data $B(x)$ shown in Fig. 3 where location $x$ was sampled on a $0.1 \mathrm{~km}$ grid over a $7 \mathrm{~km}$ by $12 \mathrm{~km}$ domain $\Omega$. This density is piecewise constant, being derived from measured prey densities (mice, ground squirrels, pocket gophers and red-backed voles) appropriate for coyotes in six different habitat types (see Ch. 7 of [11]). The main habitat feature is a strip of mesic grassland (the darkest region in the figure), which follows a valley floor and supports a high abundance of small mammals.

We use a linear relationship between prey availability and preference

$$
w(x)=1+\alpha B(x) \quad \text { for all } x \in \Omega
$$

where $\alpha$ (units of ha $/ \mathrm{kg}$ ) controls the strength of the preference per unit of prey biomass. The resulting steady-state pdfs $u^{*}$ are shown in Fig. 4 . This shows the patterns of space use for 3 different values of $\alpha$, in combination with 3 different values of length scale $L$ (20). Each column represents an $\alpha$ value, ranging from a weak preference (left column) to strong (right column). For comparison, the $w$ function is shown at the top of each column.

The computational grid was moderately-sized $(N=8591)$, the same spatial resolution as the underlying estimates of prey abundance was sampled. Once $\phi(\rho)$ had been evaluated on the grid, solving for each steady-state pdf required only $0.009 \mathrm{~s}$ using (13) with $z$ computed from $w$ by 2D FFT convolution [21]. This is 100-1000 times faster than an iterative solution for the dominant eigenvector of $K$ if neither the factorization nor analytic formula are used (a single dense matrix application of a general $K$ takes $0.45 \mathrm{~s}$ and many such iterations are required for convergence, the number depending on $L$ and the particular $w$ ). Even if the factorization (split operator method) were used to perform each iteration, our analytic formula (13) would still be 10-100 times faster.

How is steady-state space use controlled by $\alpha$ and $L$ ? Comparing the $L=6$ row (cases b,f,j) to the preference function itself (a, e, i), we see that with this large length scale $u^{*}$ is very close to proportional to $w$, as in $1 \mathrm{D}$ and as as explained in Section IA Proceeding down the figure, we see smaller $L$ values result in a $u^{*}$ with an exaggerated tendency towards space use becoming increasingly concentrated in areas of higher preference. This results in much more relative animal concentration in the mesic grassland region than would be predicted by traditional RSA. This tendency has reached its limit by the bottom $L=0.1$ row $(\mathrm{d}, \mathrm{h}, \mathrm{l})$, where $u^{*}$ is close to proportional to $w^{2}$.

Consider the right-hand column $(\mathrm{j}, \mathrm{k}, \mathrm{l})$. Changing from $L=6$ to $L=0.7$ causes a substantial increase in relative space use in the western part of the mesic grassland (see the large bump to the left in $\mathrm{k}$ ), but very little change in the eastern part of this same habitat type. The explanation is simple: the grassland strip is generally wider than $L=0.7$ on the western side resulting in a local tendency towards $u^{*} \propto w^{2}$, but narrower than $L=0.7$ on the eastern side giving here $u^{*} \propto w$. Finally in case $l L=0.1$ is narrower than all parts of the mesic grassland and the densities equalize on west and east sides. This is analogous to the transition discussed in Section IVA 1. This interesting geometric effect is absent in traditional RSA.

\section{B. Evolution in time}

In Figure 5 we show the time-dependent evolution of space use $u(x, t)$ under the master equation (2) in a single space dimension for simple piecewise linear model preference function with a single discontinuity (see panel a), with exponential kernel with length scale $L=0.01$. Zero-flux boundary conditions were created by using the nonperiodic jump kernel (19). Panels b-e show the resulting dynamics of space use, starting from an initial condition $u_{0}(x)=\delta\left(x-x_{0}\right)$ where $x_{0}=0.82$

The computation with $N=400$ grid points took 0.0005 s per time step; this was done with iterated multiplication by the dense $K$ matrix since the non-periodic choice of $\tilde{\phi}$ makes the $\Phi$ operator no longer a convolution. Notice the discontinuity feature in $u$ at $x=0.75$ establishes itself rapidly and persists throughout the full time range.

Fig. 6) shows the time-depednent evolution of space use for the 2D real-world biomass preference model (22) with $\alpha=500$ and $L=0.7$ (same parameters as Fig. $4 \mathrm{k}$ ), for a delta-function initial condition at $x_{0}=(9,4)$. After a single iteration (panel a), the local preference biases the individual's movements towards the narrow nearby eastern strip of mesic grassland. In panel (b) its space use is still split between an expanding radial distribution about its 
initial position and the nearby grassland. In panel (c), the compounded mechanistic movement steps have caused the individual's space use to become concentrated in the eastern strip of mesic grassland but the mesic grasslands in the western portion of the landscape are, at this stage, mostly unoccupied. Much later in the simulation however (panel d), the intensity of space use in the western mesic grassland areas is higher than the eastern mesic grasslands, due to the geometric effects on the steady-state $u^{*}$ described in Section IV A 2.

The simulation from which the snapshots in Figure 6 were extracted is very rapid, animating smoothly in real time at 30 frames/s even though $N \approx 10^{4}$, allowing immediate interactive model exploration. The raw calculation (no graphical animation) takes $0.008 \mathrm{~s}$ per time-step, benefitting greatly from the split operator method using FFT convolution. Performing this without the aid of the factorization (15) is about 100 times slower. Furthermore, we find that the additional effort needed to extract the mean-square displacement $\left\langle x^{2}\right\rangle(t):=\int_{\Omega}\left(x-x_{0}\right)^{2} u(x, t) d x$, a useful measure of spreading, is negligible. We remark that in order to model zero-flux boundary conditions while still using FFT convolution, a periodized kernel was used but the $w$ array was zero-padded with a border of width $O(L)$, at negligible extra cost.

\section{LOCAL BEHAVIOR NEAR A SHARP HABITAT TRANSITION}

We now examine in more detail what takes place at the discontinuities in preference function $w(x)$ that arise at the boundaries between different habitat types. As noted earlier, such discontinuities were not able to be treated in the derivation of the advection-diffusion limit in previous work [10].

For simplicity, we consider a single discontinunity on a 1D landscape, however we expect, and observe, similar behavior in the more biologically relevant case of multiple discontinuities arising at the edges of different habitat types on a 2D landscape. Consider a landscape in which there exists a single boundary between two habitat types located at $x=0$, resulting in the following discontinous preference function:

$$
w(x)= \begin{cases}1, & x<0 \\ w_{0}, & x>0\end{cases}
$$

Given a jump kernel of width $L$ it follows from (7) that $z(x)$ is a smoothed (mollified) step-function with transition region width $L$. The analytic formula (13) then tells us that the steady-state pdf jumps by a ratio of $w_{0}$ precisely at the habitat transition; however, when viewed on length scales larger than $L$, the pdf jumps by a ratio $w_{0}^{2}$. For the 1D exponential kernel (19), the analytic expression for the steady-state follows from that of $z$ via (6), and is

$$
u^{*}(x)= \begin{cases}1+\frac{W-1}{2} e^{x / L}, & x<0 \\ W\left[W-\frac{W-1}{2} e^{-x / L}\right], & x>0\end{cases}
$$

The spatial decay length is thus the same as that of the kernel $\phi$. This is shown in Fig. 77a.

How fast is this equilibrium reached? We demonstrate in Fig. 7b that the transition region's shape reaches its approximate equilibrium in only a single time-step (a slight change also occurs over the next few time-steps). Here we chose initial conditions which already matched the global pdf ratio of $w_{0}^{2}$, in order to study the equilibration in this local region alone.

\section{A. Effective boundary condition for advection-diffusion equation}

Armed with this understanding of the local behavior at a preference function discontinuity, we can incorporate this into the Fokker-Planck PDE model for the evolution of $u(x, t)$ in the $\tau \rightarrow 0$ limit (in which case kernel width $L$ must also go to zero at an appropriate rate). We remind the reader that the Fokker-Planck equation is

$$
\frac{\partial}{\partial t} u=-\frac{\partial}{\partial x}[c(x) u]+\frac{\partial^{2}}{\partial x^{2}}[d(x) u]
$$

where $c(x)$ and $d(x)$, representing drift and diffusion rates, take on values given by the $\tau$-scaling of the second moment of the jump kernel $\phi[2,10,11]$

We combine two observations: i) locally the steady-state in the vicinity of a discontinuity in $w$ enforces a multiplicative jump (the square of the $w$ ratio) in $u$, and ii) in the $\tau \rightarrow 0$ limit the Fokker-Planck equation evolves on much slower time-scales than the local equilibration in this vicinity (which happens in $O(\tau)$ ). Thus we expect that, 
for evolution on time-scales long relative to $\tau$ the transition region is in local equilibrium, with the effective boundary condition

$$
\frac{u\left(x_{-}, t\right)}{\left(w_{-}\right)^{2}}=\frac{u\left(x_{+}, t\right)}{\left(w_{+}\right)^{2}}, \quad \text { for all } t>0
$$

where the subscripts - and + indicate limiting values on the left and right side of the discontinuity respectively. Similarly, by conservation of the flux $J(x)=\frac{\partial}{\partial x}[d(x) u]-c(x) u$ across the discontinuity, we must have that if $d(x)$ is continuous and $c(x)=0$ then

$$
\frac{\partial}{\partial x} u\left(x_{-}, t\right)=\frac{\partial}{\partial x} u\left(x_{+}, t\right), \quad \text { for all } t>0,
$$

at a step discontinuity in $w$. We may now interpret (26) and (27) as matching conditions for coupled advectiondiffusion equations on either side of the discontinuity. In this way we have a recipe to understand the diffusion limit even in the presence of discontinuous preference functions. We remark that our assumption of locally vanishing $c$ corresponds to no gradient in $w$ locally on either side (the case with general values of $w^{\prime}$ either side we postpone for future work). The above argument is non-rigorous, relying on reasoning based on separation of length- and time-scales. However it seems to be supported qualitatively by the evidence in Fig. 5b-e (although at early times (26) does not appear to hold accurately). We suggest that a more detailed analysis via matched asymptotic expansions should be carried out.

\section{CONCLUSIONS}

We have analyzed the mathematical properties of a mechanistic resource selection (MERSA) model that captures the influence of spatially-localized habitat preference on the movement behavior of individuals and predicts their resulting patterns of space use. The model combines random foraging motion with a local sensitivity to habitat preference over a 'perceptual radius' $L$. Directed movement bias is generated in a similar manner to the angle-biased (von Mises) jump kernels used in [11], and also becomes equivalent in the small- $L$ limit to continuous-time advectiondiffusion in a 'confining potential' as in [3]. Our analysis shows that the model has a desirable factorization (15) which yields a simple closed-form formula (13) for the steady state pdf. The effect of the compounded random movement decisions upon this steady state pdf is a novel geometry-dependent scaling with the preference function: linear when $L$ is large (compared to local habitat features), but quadratic when $L$ is small.

These novel spatial effects are absent in conventional RSA, and have only been analysed previously in mechanistic home range models in the context of the advection-diffusion limit [10] in which the perceptual radius of individuals is small $(L \rightarrow 0)$. Our analytic formula developed here allows this to be understood for the case of discontinuous preference functions and for any given perceptual radius $L$.

Large gains in computational efficiency have been demonstrated throughout, including the case of discontinuous 2D preference functions motivated by observations of spatially-varying prey availability across different habitat types. We believe such efficient forward models will be important tools, as inverse modeling, and fitting of multiple model parameters to observations, become more popular and time-consuming.

Although we did not construct or study them numerically in this work, we expect that the benefits demonstrated here for a model that is linear in $u$ (with prey- or spatially- dependent movement rates) will also apply in the analysis of non-linear models such as those with density-dependent diffusion (e.g. Sec. 3 of [18]), and scent-mediated interactions between multiple animal packs [6, 11, 18]. For example, chemotaxis could be included in the preference function $w$, in which case our analytic formula (13) could be used to turn a coupled PDE system into coupled algebraic systems, a huge simplification. We also expect that by extending our preliminary operator analysis (Proposition 2), the spectral properties, and hence equilibration rates of animal home range space use, may be deduced.

Concise Matlab codes for computation of all figures in this work are freely available at http://math. dartmouth.edu/ ahb/moorcroft/

\section{Acknowledgments}

AHB and PRM thank Robert L. Crabtree for the dataset on small mammal abundance in Yellowstone National Park. AHB thanks Jonathan Goodman and Luc Rey-Bellet for useful discussions on the properties of Markov operators. 
AHB is partially funded by NSF-0507614.

[1] M. S. Boyce and L. L. McDonald. Trends in Ecology and Evolution, 14:268-272, 1999.

[2] C. W. Gardiner. Handbook of stochastic methods for physics, chemistry, and the natural sciences, 3rd ed., volume 13 of Springer Series in Synergetics. Springer-Verlag, Berlin, 2004.

[3] L. Giuggioli, G. Abramson, V. M. Kenkre, R. R. Parmenter, and T. L. Yates. Theory of home range estimation from displacement measurements of animal populations. J. Theor. Biol., 240:126-135, 2006.

[4] D. A. Johnson. The comparison of usage and availability measurementts for evaluating resource preference. 61:65-71.

[5] P. Kareiva. Experimental and mathematical analyses of herbivore movement: Quantifying the influence of plant spacing and quality on foraging discrimination. Ecological Monographs, 52:261-282, 1982.

[6] M. A. Lewis, K. A. J. White, and J. D. Murray. Analysis of a model for wolf territories. J. Math. Biol., 35:749-774, 1997.

[7] B. F. J. Manly, L. L. McDonald, and D. L. Thomas. Resouce selection by animals: statistical design and analysis for field studies. Chapman and Hall, 1993.

[8] S. P. Meyn and R. L. Tweedie. Markov Chains and Stochastic Stability. Springer-Verlag, London, 1993.

[9] P. R. Moorcroft. Territoriality and carnivore home ranges. PhD thesis, Dept. of Ecology and Evolutionary Biology, Princeton University, 1997.

[10] P. R. Moorcroft and A. H. Barnett. Mechanistic home range models and resource selection analysis: a reconciliation and unification, 2006. submitted.

[11] P. R. Moorcroft and M. A. Lewis. Mechanistic home range analysis. Monographs in population biology. Princeton University Press, Princeton, NJ, 2006.

[12] P. R. Moorcroft, M. A. Lewis, and R. L. Crabtree. 2006.

[13] A. Okubo. Diffusion and ecological problems. Springer-Verlag, 1980.

[14] H. Othmer, S. R. Dunbar, and W. Alt. Models of dispersion in biological systems. J. Math. Biol., 26:263-298, 1988.

[15] C. Patlak. Random walk with persistence and external bias. Bull. Math. Biophys., 15:311-338, 1953.

[16] W. H. Press, S. A. Teukolsky, W. T. Vetterling, and B. P. Flannery. Numerical recipes in C. Cambridge University Press, Cambridge, 2002.

[17] P. Turchin. Translating foraging movements in heterogeneous environments into the spatial distribution of foragers. Ecology, 72:1253-1266, 1991.

[18] K. A. J. White, M. A. Lewis, and J. D. Murray. A model for wolf-pack territory formation and maintenance. J. Theor. Biol., 178:26-43, 1996.

[19] One may ask whether discrete or continuous time is more appropriate for animal movement modeling. Clearly in reality animals move in continuous time, however a continuous-time diffusion equation (Brownian process) cannot be realistic on the shortest time-scales for the simple reason that this would require infinite movement speed (e.g. see [14]). Therefore models with a fixed discrete time step remain crucial for fine-scale modeling.

[20] Note that in the stochastic literature the order of $x$ and $x^{\prime}$ is often reversed [2]

[21] We have also tried other model preference functions on larger grids: a 200 by 200 grid $(N=40000)$ requires 0.025 to find $u^{*}$. By contrast, note that solving for $u^{*}$ without the factorization of $K$ is impractical (even representing $K$ as a dense $N$-by- $N$ matrix on this grid would require 12 GB of memory). 

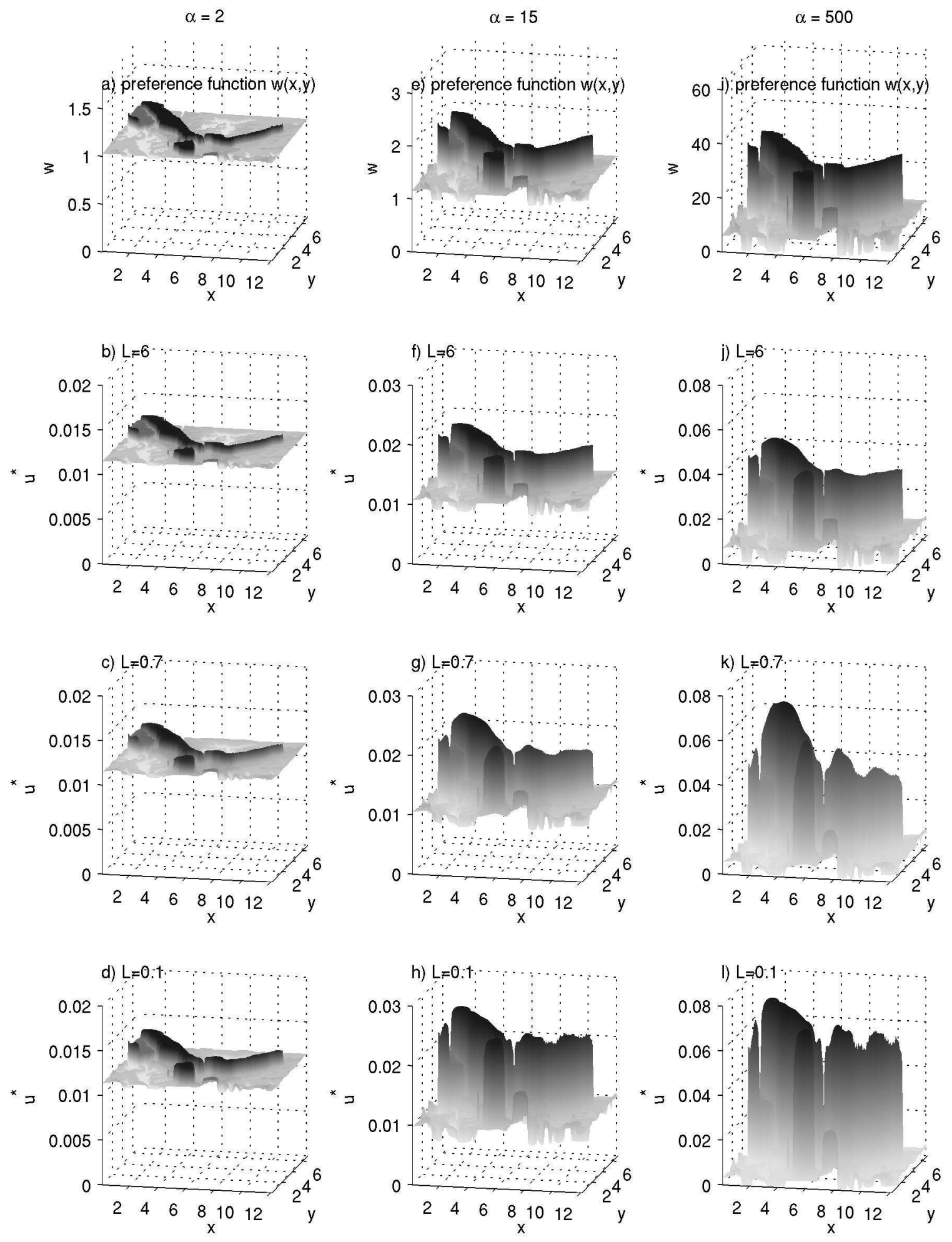

FIG. 4: Steady-state 2D pdfs $u^{*}$ for the exponential jump kernel (20), applied to preference functions $w$ derived from biomass data shown in Fig. 3 The three columns represent the choices $\alpha=2,15,500$ in the preference model (22). At the top of each column is a surface plot of the preference function, followed below by surface plots of $u^{*}$ for three decreasing values of $L$. 
a)

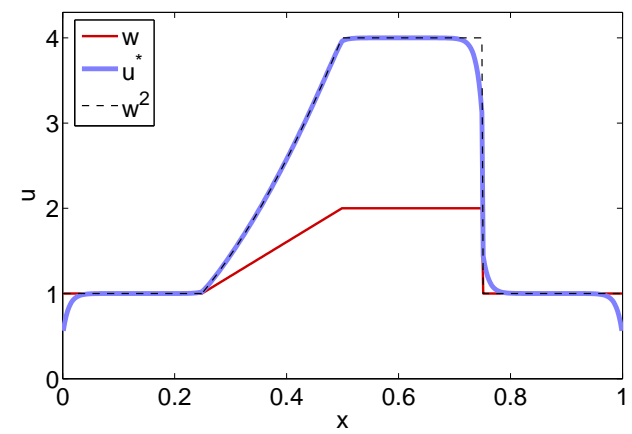

b) $t / \tau=3$

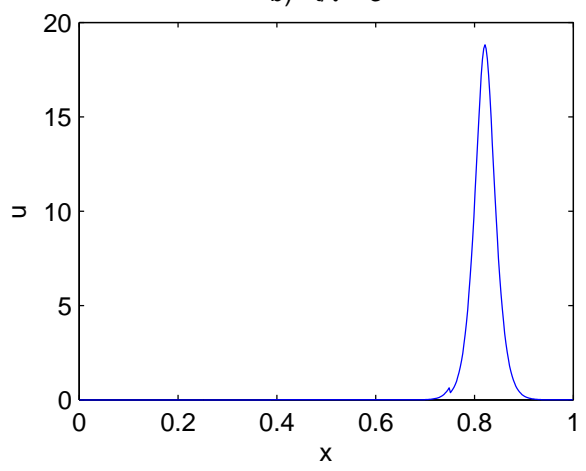

d) $t / \tau=200$

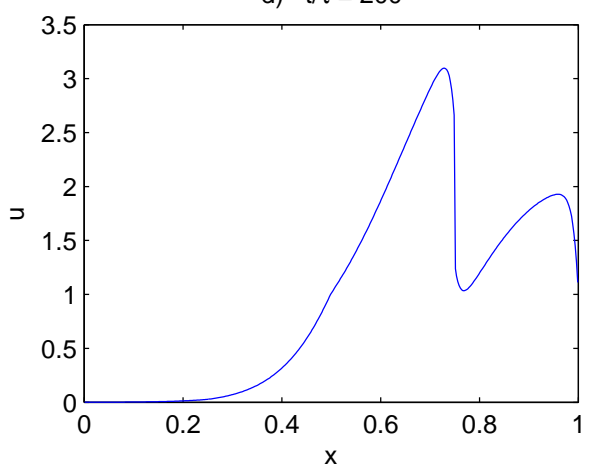

c) $t / \tau=30$

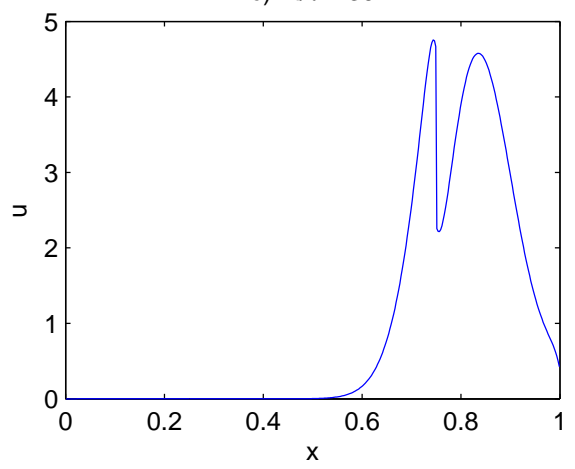

e) $t / \tau=1500$

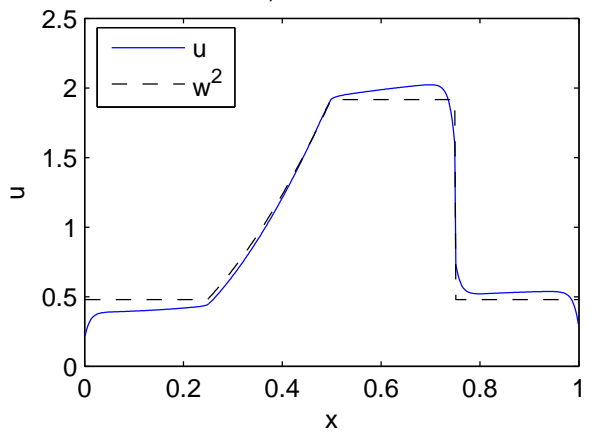

FIG. 5: a) Piecewise linear model preference function $w(x)$, steady state pdf $u^{*}(x)$, and predicted limiting case of steady-state pdf (11) (dashed). b)-e) Snapshots of time evolution of $u(x, t)$ under master equation (2) in 1D, at four times (indicated by $t / \tau$ the number of iterations). The exponential jump pdf of (19) is used with $L=0.01$. In e) $u$ is very close to steady-state; the prediction (11) is also shown (dashed). 
a) $t / \tau=1$

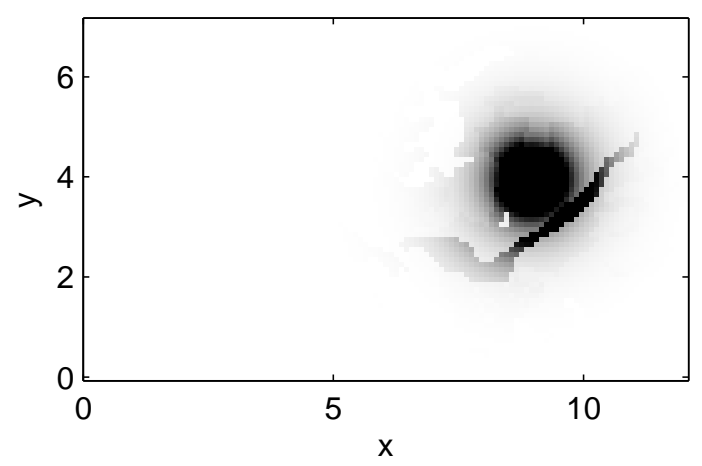

c) $t / \tau=20$

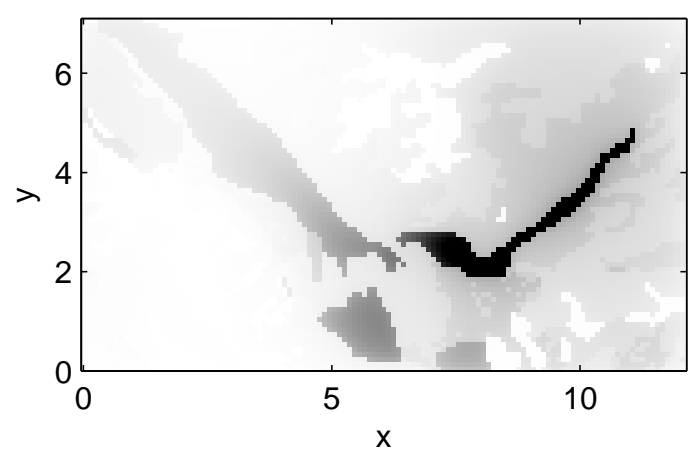

b) $t / \tau=4$

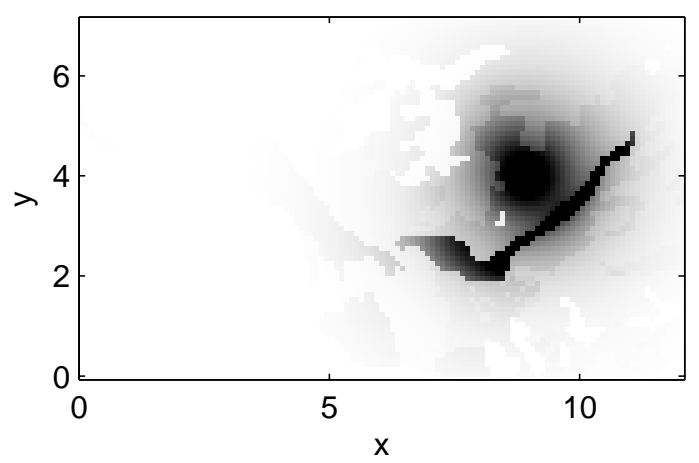

d) $t / \tau=200$

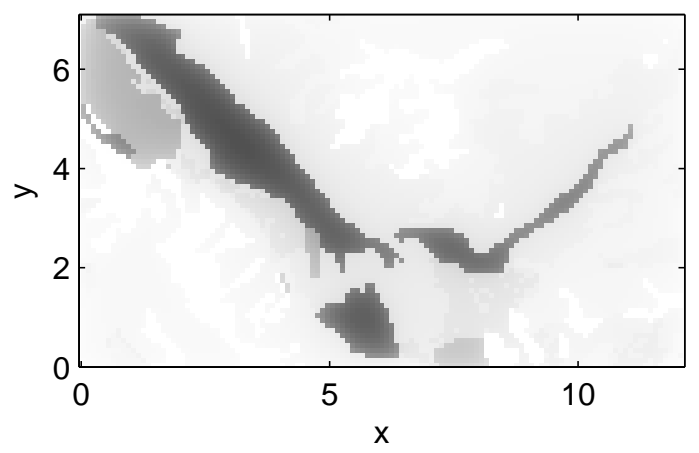

FIG. 6: Density plots snapshots of the time evolution of $u(x, t)$ under master equation (2) in 2D, at four times (indicated by $t / \tau$ the number of iterations). The exponential jump pdf of (20) is used with $L=0.7$, and preference function derived from biomass data in Fig. 36via Eq.(22) with $\alpha=500$. In d) $u$ is very close to steady-state, which is shown in Fig. [4k.

a)

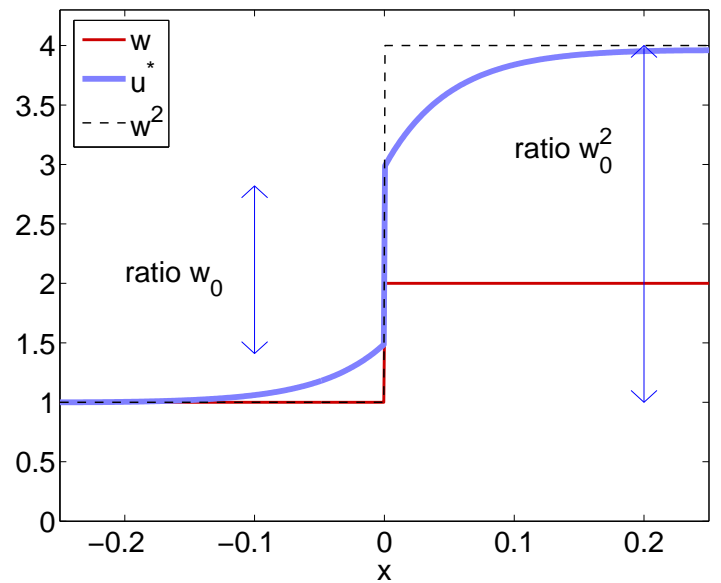

b)

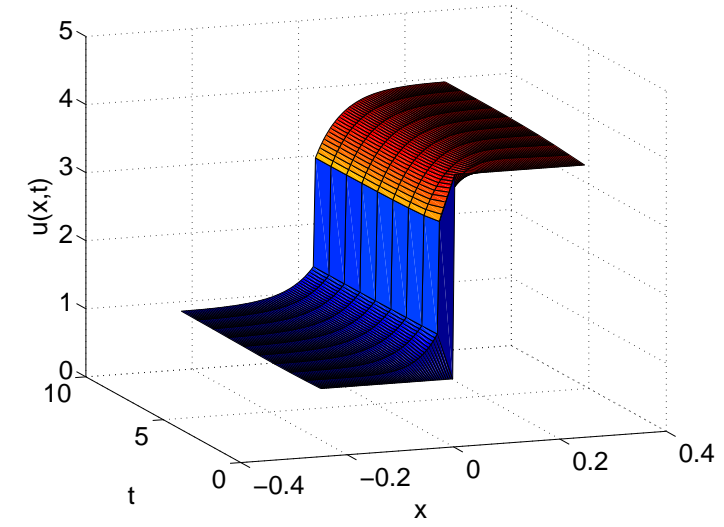

FIG. 7: a) Detail of transition in steady-state space use occurring within distance $L$ of a discontinuity in preference function of the form (23) with $w_{0}=2$, for the $1 \mathrm{D}$ exponential jump kernel of (19). b) Time-evolution of $u(x, t)$ in this same local region for 10 time-steps of the master equation (2), starting from an initial pdf $u_{0}(x)=w(x)^{2}$. 